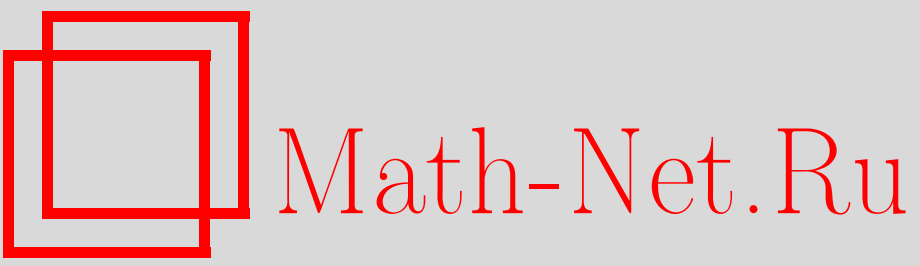

И. З. Голубчик, В. В. Соколов, Интегрируемые уравнения на $\mathbb{Z}$-градуированных алгебрах Ли, ТМФ, 1997, том 112, номер 3, 375-383

DOI: https://doi.org/10.4213/tmf1049

Использование Общероссийского математического портала Math-Net.Ru подразумевает, что вы прочитали и согласны с пользовательским соглашением

http://www.mathnet.ru/rus/agreement

Параметры загрузки:

IP : 54.224 .60 .19

26 апреля 2023 г., 13:35:34 


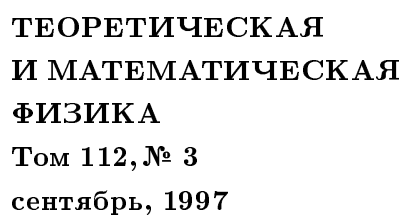

И.З. Голубчик* , В.В. Соколов*

\section{ИНТЕГРИРУЕМЫЕ УРАВНЕНИЯ}

\section{НА Z्Z-ГРАДУИРОВАННЫХ АЛГЕБРАХ ЛИ}

Рассматриваются эволюционные системы, обладающие $L$ - $A$-парами в $\mathbb{Z}$-градуированных алгебрах Ли. При этом с одним и тем же оператором $L$ связываются несколько разных иерархий интегрируемых систем, соответствующих разным разложениям нулевой компоненты $\mathbb{Z}$-градуированной алгебры в прямую сумму двух подалгебр. Это позволяет построить новые примеры многокомпонентных интегрируемых систем типа уравнений Бюргерса, мКдФ, НУШ и Буссинеска.

В работе [1] для оператора

$$
L=D_{x}+a \lambda+q(x, t)
$$

где $\lambda$ - спектральный параметр, $q$ принадлежит алгебре Ли $\mathfrak{G}, a$ - постоянный элемент $\mathfrak{G}$, приведен один из способов построения оператора $A=\sum_{i=0}^{n} p_{i} \lambda^{i}$ такого, что операторное соотношение $L_{t}=[A, L]$ эквивалентно эволюционному уравнению вида

$$
q_{t}=F\left(q, q_{x}, q_{x x}, \ldots\right) .
$$

Этот способ основан на приведении оператора $L$ к диагональному виду и позволяет, кроме оператора $A$, конструктивно строить также высшие симметрии и законы сохранения для уравнения (2).

Конструкция из работы [1] легко обобшается на операторы $L$ более обшего вида:

$$
L=D_{x}+a \lambda^{n+1}+\sum_{i=-m}^{n} q_{i}(x, t) \lambda^{i} .
$$

В данной работе при построении оператора $A$ вместо использованного в [1] стандартного разложения алгебры Ли рядов Лорана $\mathfrak{G}\left[\left[\lambda, \lambda^{-1}\right]\right]$ в сумму полиномов от $\lambda$ и рядов, содержаших только отрицательные степени $\lambda$, мы пользуемся более сложной конструкцией, связанной с разложением алгебры $\mathfrak{G}$ в прямую сумму подпространств, каждое из которых является подалгеброй в $\mathfrak{G}$. Это позволяет для одного и того же оператора $L$ построить несколько различных операторов $A$ и, таким образом, значительно расширить список интегрируемых уравнений (2).

Для того чтобы включить в рассмотрение уравнения над алгебрами Каца-Муди [1], а также матричные и векторные интегрируемые системы, рассмотренные в [2-9], вместо

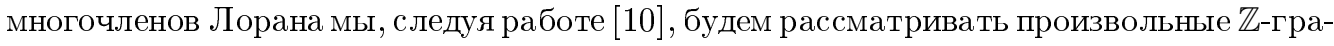
дуированные алгебры Ли.

* Математический институт Уфимского научного центра РАН, Уфа, Россия. E-mail: sokolov@imat.rb.ru 
1. Общая схема. Напомним, что алгебра Ли $\mathfrak{G}=\bigoplus_{i \in \mathbb{Z}} \mathfrak{G}_{i}$ называется $\mathbb{Z}$-градуированной, если $\mathfrak{G}_{i}-$ векторные подпространства такие, что $\left[\mathfrak{G}_{k}, \mathfrak{G}_{j}\right] \subseteq \mathfrak{G}_{k+j}$. Ясно, что $\mathfrak{G}_{0}$ является подалгеброй в $\mathfrak{G}$.

Предположим, что

$$
\mathfrak{G}_{0}=\mathfrak{A}_{+} \oplus \mathfrak{A}_{-}
$$

есть прямая сумма векторных подпространств, являюшихся подалгебрами Ли. Если $\mathfrak{G}_{0}$ - полная матричная алгебра, то наиболее стандартными являются ее разложения в сумму верхнетреугольных и нижнетреугольных, а также верхнетреугольных и кососимметрических матрищ.

В приведенных ниже примерах $\mathfrak{G}_{0}$ - алгебра всех диагональных матриц с элементами из произвольного ассоциативного кольца $\mathfrak{K}$. Соответствующее уравнение (2) представляет собой одно или несколько уравнений, в которых неизвестные функции принимают значения в $\mathfrak{K}$. Мы будем называть такие уравнения неабелевыми. Конечно, координатная запись неабелева уравнения приводит к обычной многокомпонентной системе эволюционных уравнений.

Если $\mathfrak{K}$ - полная матричная алгебра $g l(k)$, то в наших примерах $\mathfrak{G}_{0}-$ множество блочно-диагональных матриц. В простейшем случае двух блоков задача разложения $\mathfrak{G}_{0}$ в прямую сумму своих подалгебр самым тесным образом связана с постоянными решениями классического модифицированного уравнения Янга-Бакстера (см. $[11,12])$ на $g l(k)$ :

$$
R([R(X), Y]-[R(Y), X])=[X, Y]+[R(X), R(Y)], \quad X, Y \in g l(k) .
$$

А именно, для любого оператора $R$, удовлетворяюшего (4),

$$
\mathfrak{A}_{-}=\left\{\left(\begin{array}{ll}
a & 0 \\
0 & a
\end{array}\right)\right\} \quad \text { и } \quad \mathfrak{A}_{+}=\left\{\left(\begin{array}{cc}
a+R(a) & 0 \\
0 & -a+R(a)
\end{array}\right)\right\}
$$

задают искомое разложение.

Пусть элемент $\alpha \in \mathfrak{G}_{1}$ удовлетворяет условию

$$
\operatorname{Ker}(\operatorname{ad} \alpha) \oplus \operatorname{Im}(\operatorname{ad} \alpha)=\mathfrak{G} .
$$

Рассмотрим оператор $L$ вида

$$
L=D_{x}+\alpha+q(x, t), \quad q \in \mathfrak{G}_{0} .
$$

Обозначим через $\overline{\mathfrak{G}}$ алгебру Ли $\left\{\sum_{i=-\infty}^{k} g_{i} \mid k \in \mathbb{Z}, g_{i} \in \mathfrak{G}_{i}\right\}$. Будем называть элементы $\overline{\mathfrak{G}}$ рядами.

ПРЕДЛОЖЕнИЕ 1. Существуют единственнъе рядъ $и=\sum_{i=-\infty}^{-1} u_{i}$, $u_{i} \in \operatorname{Im}(\operatorname{ad} \alpha) \cap \mathfrak{G}_{i}, u h=\sum_{i=-\infty}^{0} h_{i}, \quad h_{i} \in \operatorname{Ker}(\operatorname{ad} \alpha) \cap \mathfrak{G}_{i}$, maкue, ито

$$
\exp (\operatorname{ad} u)(L)=D_{x}+\alpha+h
$$


ЗАМЕчАниЕ 1 . Элементы $u_{i}$ и $h_{i}$ являются дифференциальными многочленами от $q$. Более точно, для любого базиса в $\mathfrak{G}$ коэффициенты в разложении $u_{i}$ и $h_{i}$ по этому базису - многочлены от коэффициента $q$ и его производных по $x$.

Предположим, что элемент $\alpha$, фигурируюший в операторе (6), связан с разложением (3) условием

$$
\mathfrak{A}_{-} \subseteq \operatorname{Ker}(\operatorname{ad} \alpha) .
$$

Отметим, что, имея одно разложение (3), удовлетворяюшее условию (8), можно получить семейство других, сопрягая его произвольным элементом из группы Ли алгебры Ли $\operatorname{Ker}(\operatorname{ad} \alpha) \cap \mathfrak{G}_{0}$.

Положим

$$
\begin{aligned}
& \overline{\mathfrak{G}}_{+}=\bigoplus_{i>0} \mathfrak{G}_{i}+\mathfrak{A}_{+}, \\
& \overline{\mathfrak{G}}_{-}=\sum_{i=-\infty}^{-1} \mathfrak{G}_{i}+\mathfrak{A}_{-} .
\end{aligned}
$$

ПРЕДЛОЖЕНИЕ 2. Пусть $\beta$ - постоянный әлемент из иентра подалгебры $\operatorname{Ker}(\operatorname{ad} \alpha)$,

$$
A_{\beta}=(\exp (-\operatorname{ad} u)(\beta))_{+},
$$

где "+" отмечает проекцию на $\overline{\mathfrak{G}}_{+}$параллельно $\overline{\mathfrak{G}}_{-}$. Тогда $\left[A_{\beta}, L\right] \in \sum_{-m}^{n} \mathfrak{G}_{i}$.

ЗАмЕчАниЕ 2. Утверждение предложения 2 означает, что соотношение $L_{t}=\left[A_{\beta}, L\right]$ эквивалентно некоторой эволюционной системе относительно неизвестной $q(x, t)$. Нетрудно проверить, что в простейшем случае $\mathfrak{A}_{+}=\mathfrak{G}_{0}, \mathfrak{A}_{-}=\{0\}$ правая часть уравнения для $q$ лежит в $\operatorname{Im}(\operatorname{ad} \alpha)$, и можно считать, что $q(x, t) \in \operatorname{Im}(\operatorname{ad} \alpha)$. Кроме того, всегда допускается редукция $q \in \mathfrak{A}_{+}$.

Следуюшие утверждения, которые доказьваются примерно так же, как и соответствуюшие теоремы из [1], показывают, что построенные эволюционные системы обладают высшими симметриями и законами сохранения.

Теорема 1. Пусть $\beta$ и $\gamma-$ произвольные әлементы центра подалгебры $\operatorname{Ker}(\operatorname{ad} \alpha)$. Тогда потоки $L_{t}=\left[A_{\beta}, L\right]$ и $L_{\tau}=\left[A_{\gamma}, L\right]$ коммутируют между собой.

Известно, что законы сохранения для уравнений (2) тесно связаны с инвариантными формами на $\overline{\mathfrak{G}}$. Напомним, что билинейная форма $\langle X, Y\rangle$ с числовыми значениями называется инвариантной, если $\langle[X, Y], Z\rangle=-\langle Y,[X, Z]\rangle$ для всех $X, Y, Z \in \overline{\mathfrak{G}}$. Будем считать, что форма согласована с градуировкой, т.е.

$$
\left\langle\sum X_{i}, \sum Y_{i}\right\rangle=\sum\left\langle X_{i}, Y_{-i}\right\rangle
$$

Заметим, что сумма в правой части всегда конечна.

Tеорема 2. Пусть $\langle X, Y\rangle$ - инвариантная форма на $\overline{\mathfrak{G}}$ и $\beta, \gamma-$ әлементы из центра подалгебры Ли $\operatorname{Ker}(\operatorname{ad} \alpha)$. Тогда $\rho=\langle h, \gamma\rangle$, где $h-$ ряд из предложения 1 , является плотностью закона сохранения для уравнения Лакса $L_{t}=\left[A_{\beta}, L\right]$. 
2. Примеры интегрируемых эволюционных систем, порожденных градуированными алгебрами Ли. Один из наиболее интересных классов $\mathbb{Z}$-градуированных алгебр Ли - это так называемые алгебры Каца-Муди. Напомним определения. Пусть $\mathfrak{B}$ - полупростая алгебра Ли и $f$ - автоморфизм конечного порядка $m$ алгебры Ли $\mathfrak{B}$. Положим

$$
\mathfrak{G}_{i}=\left\{a \in \mathfrak{B} \mid f(a)=\mu^{i} a\right\} \lambda^{i},
$$

где $\mu$ - первообразный корень $m$-й степени из 1 . Тогда алгебра Ли $\mathfrak{G}=\bigoplus_{i \in \mathbb{Z}} \mathfrak{G}_{i} \mathbb{Z}$-градуирована. Она называется алгеброй Каца-Муди. В отличие от [1] в настоящей работе основными для нас являются примеры, когда $\mathfrak{G}_{0}-$ некоммутативная подалгебра в $\mathfrak{G}$.

ПРИМЕР 1. Системы типа модифицированного уравнения Кортевега-де Фриза. Пусть $\mathfrak{B}$ - алгебра матриц второго порядка над ассоциативным кольцом $\mathfrak{K}$ с единицей, $f$-внутренний автоморфизм, задаваемьй матрицей $\left(\begin{array}{cc}1 & 0 \\ 0 & -1\end{array}\right)$. Легко видеть, что $\mathfrak{G}_{2 i}-$ множество всех диагональных матриц, а $\mathfrak{G}_{2 i+1}-$ множество всех матриц с нулевой диагональю. Оператор $L$ задан формулой $(1)$, где $a=\left(\begin{array}{ll}0 & 1 \\ 1 & 0\end{array}\right)$ и $q \in \mathfrak{G}_{0}$. Оператор $A=A_{\beta}$ определяется формулой (11), где $\beta=\left(\begin{array}{ll}0 & 1 \\ 1 & 0\end{array}\right) \lambda^{3}$.

Различным разложениям (3) с условием $\mathfrak{A}_{-} \subseteq\left\{\left(\begin{array}{ll}a & 0 \\ 0 & a\end{array}\right)\right\}$ соответствуют различные уравнения (2). Нетрудно проверить, что в случае $\mathfrak{A}_{+}=\mathfrak{G}_{0}, \mathfrak{A}_{-}=\{0\}$ уравнение $(2)$ для $q=\left(\begin{array}{cc}a & 0 \\ 0 & -a\end{array}\right)$ есть стандартное неабелево модифицированное уравнение Кортевега-де Фриза

$$
a_{t}=a_{x x x}+3 a^{2} a_{x}+3 a_{x} a^{2}, \quad a \in \mathfrak{K} .
$$

Рассмотрим теперь случай, когда $\mathfrak{A}_{-}=\left\{\left(\begin{array}{ll}a & 0 \\ 0 & a\end{array}\right)\right\}$. Легко проверить, что тогда в качестве $\mathfrak{A}_{+}$можно взять

$$
\mathfrak{A}_{+}=\left\{\left(\begin{array}{cc}
a+R(a) & 0 \\
0 & -a+R(a)
\end{array}\right)\right\}
$$

где оператор $R: \mathfrak{K} \rightarrow \mathfrak{K}$ удовлетворяет модифицированному уравнению Янга-Бакстера на ассоциативном кольце

$$
R([R(X), Y]-[R(Y), X])=[X, Y]+[R(X), R(Y)], \quad X, Y \in \mathfrak{K} .
$$

Простейшими решениями уравнения (12) являются $R=1$ и $R=-1$. Эти решения допускают следуюшие обобщения. Пусть оператор $T \in \mathfrak{K}$ таков, что $T^{2}=1$. Тогда оператор $R(X)=T X$ удовлетворяет (12). Если $e$ - идемпотент (т.е. $e^{2}=e$ ), то оператор $R(X)=X-2 e X(1-e)$ также является решением $(12)$.

Соответствуюшее уравнение $(2)$ на функцию $q \in \mathfrak{G}_{0}$ представляет собой эволюционную неабелеву систему вида

$$
\begin{aligned}
a_{t} & =-P_{x}+[R(P), a]+[P, b], \\
b_{t} & =-R\left(P_{x}\right)+[P, a]+[R(P), b],
\end{aligned}
$$


где $a, b \in \mathfrak{K}$ и

$$
P=a_{x x}+2\left[b, a_{x}\right]+\left[b_{x}, a\right]+[b,[b, a]]-2 a^{3} .
$$

Легко видеть, что центр $\operatorname{Ker}(\operatorname{ad} \alpha)$ состоит из различных степеней $\alpha$, причем четные степени приналежат центру алгебры $\mathfrak{G}$ и поэтому приводят к нулевой симметрии. Таким образом, система (13) обладает высшими симметриями всех нечетных порядков. В частности, симметрия первого порядка имеет вид

$$
\begin{aligned}
a_{\tau} & =-a_{x}+[R(a), a]+[a, b], \\
b_{\tau} & =-R\left(a_{x}\right)+[R(a), b] .
\end{aligned}
$$

Отметим, что система (14) встречается в работе [13], посвященной обобщениям метода факторизации.

Согласно замечанию 2 система (13) и все ее симметрии допускают редукцию на $\mathfrak{A}_{+}$, которая описывается формулой $b=R(a)$. В результате получается система

$$
a_{t}=-P_{x}+P * a-a * P, \quad a \in \mathfrak{K},
$$

где $P=a_{x x}+2 a * a_{x}+a_{x} * a+a *(a * a)-2 a^{3}$. Здесь $a^{3}-$ ассоциативный куб в $\mathfrak{K}$,

$$
X * Y=[R(X), Y] .
$$

Алгебраические свойства операции (16) обсуждались в [14].

В случае $R=1$ уравнение (15) приобретает вид

$$
a_{t}=-a_{x x x}+3\left(a_{x x} a-a a_{x x}\right)+6 a a_{x} a .
$$

Для $\mathfrak{K}$, совпадаюшего с полной матричной алгеброй, это уравнение с точностью до замены $t \rightarrow-t$ появлялось в [15].

Для более обшего решения уравнения Янга-Бакстера $R(X)=T X, T^{2}=1$ уравнение (15) имеет вид

$$
\begin{aligned}
a_{t}=a_{x x x} & +3 T a a_{x x}-3 a_{x x} T a+3 T a_{x}^{2}-3 a_{x} T a_{x}-3 a a_{x} a- \\
& -3 a_{x} a^{2}+T a T a a_{x}+T a T a_{x} a-T a^{2} T a_{x}-4 T a a_{x} T a+T a_{x} T a^{2}- \\
& -T a_{x} a T a+3 a_{x} T a T a-a T a^{3}-a^{2} T a^{2}+2 a^{3} T a-2 T a T a T a^{2}+ \\
& +T a T a^{2} T a+T a^{2} T a T a .
\end{aligned}
$$

ПримеР 2. Системы типа нелинейного уравнения Шредингера. В этом примере $\mathfrak{B}$ - 3-градуированная алгебра Ли

$$
\mathfrak{B}=\mathfrak{B}_{-1} \oplus \mathfrak{B}_{0} \oplus \mathfrak{B}_{1},
$$

т.е. $\mathbb{Z}$-градуированная алгебра Ли, в которой все компоненты, кроме $\mathfrak{B}_{-1}, \mathfrak{B}_{0}, \mathfrak{B}_{1}$, являются нулевыми. Такие алгебры Ли тесно связаны с йордановыми парами [16].

Простейший пример 3-градуированной алгебры Ли - множество $2 \times 2$-матрищ над ассоциативным кольцом $\mathfrak{K}$, где

$$
\mathfrak{B}_{0}=\left\{\left(\begin{array}{ll}
a & 0 \\
0 & b
\end{array}\right)\right\}, \quad \mathfrak{B}_{1}=\left\{\left(\begin{array}{ll}
0 & c \\
0 & 0
\end{array}\right)\right\}, \quad \mathfrak{B}_{-1}=\left\{\left(\begin{array}{ll}
0 & 0 \\
d & 0
\end{array}\right)\right\} .
$$


Широкий класс 3-градуированных алгебр Ли - это суперструктурные алгебры йордановых тройных систем [16].

В качестве $\mathfrak{G}$ рассматривается алгебра многочленов Лорана $\mathfrak{B}\left[\lambda, \lambda^{-1}\right]$ над $\mathfrak{B}$. Ясно, что если положить $\mathfrak{G}_{i}=\mathfrak{B} \lambda^{i}$, то алгебра Ли $\mathfrak{G}=\bigoplus_{i \in \mathbb{Z}} \mathfrak{G}_{i}$ становится $\mathbb{Z}$-градуированной.

Без ограничения обшности можно считать, что $\mathfrak{B}_{0}$ содержит элемент $a$ такой, что $\left[a, b_{1}+b_{0}+b_{-1}\right]=b_{1}-b_{-1}$ для любых $b_{i} \in \mathfrak{B}_{i}$. Положим $\alpha=a \lambda$. Тогда $\operatorname{Ker}(\operatorname{ad} \alpha)$ есть $\mathfrak{B}_{0}\left[\lambda, \lambda^{-1}\right]$. Следовательно, для любого $b$ из центра $\mathfrak{B}_{0}$ элемент $\beta=b \lambda^{2}$ лежит в $\operatorname{Ker}(\operatorname{ad} \alpha)$.

В случае $b=a$ и $\mathfrak{A}_{+}=\mathfrak{G}_{0}, \mathfrak{A}_{-}=\{0\}$ соответствуюшая эволюционная система имеет вид (ср. [2])

$$
u_{t}=u_{x x}+[[v, u], u], \quad v_{t}=-v_{x x}-[[u, v], v]
$$

где $u \in \mathfrak{B}_{-1}, v \in \mathfrak{B}_{1}$. Эта система является перезаписью нелинейного уравнения Шредингера на йордановых парах, рассмотренного в [6]. Согласно теореме 1 система (21) имеет высшие симметрии всех порядков.

В случае алгебры (20) система (21) есть обычное неабелево (например, матричное) нелинейное уравнение Шредингера

$$
u_{t}=u_{x x}-2 u v u, \quad v_{t}=-v_{x x}+2 v u v .
$$

Рассмотрим теперь случай $b=a$ и $\mathfrak{A}_{-}=\mathfrak{B}_{0}$,

$$
\mathfrak{A}_{+}=\left\{q+S(q) \mid q \in \mathfrak{B}_{1} \oplus \mathfrak{B}_{-1}\right\}
$$

где $S$ - линейный оператор из $\mathfrak{B}_{1} \oplus \mathfrak{B}_{-1}$ в $\mathfrak{B}_{0}$ такой, что $(22)$ - подалгебра Ли. Соответствуюшая система для $q \in \mathfrak{B}_{-1} \oplus \mathfrak{B}_{1}$ (см. замечание 2 ) имеет вид

$$
\begin{aligned}
q_{t}=\left[a, q_{x x}\right]-\left[a, q_{x}\right] * q+q *\left[a, q_{x}\right]+q_{x} *[a, q]+ \\
+q *\left[a, q_{x}\right]-(q *[a, q]) * q+q *(q *[a, q]),
\end{aligned}
$$

где $X * Y=[S(X), Y]$

Если алгебра $\mathfrak{B}$ задается формулой $(20)$, то в качестве $S$ можно взять отображение

$$
S\left(\begin{array}{ll}
0 & c \\
d & 0
\end{array}\right)=\frac{1}{2}\left(\begin{array}{cc}
d-c+R(d+c) & 0 \\
0 & c-d+R(d+c)
\end{array}\right),
$$

где $R$ - любое решение модифишированного уравнения Янга-Бакстера (12).

В частности, если $R=1$, то уравнение (23) имеет вид

$$
\begin{aligned}
& c_{t}=c_{x x}+2 d c_{x}+2 d_{x} c-2 c_{x} c+2 c d c-2 d c^{2} \\
& d_{t}=-d_{x x}-2 c d_{x}-2 c_{x} d+2 d_{x} d-2 d c d+2 c d^{2} .
\end{aligned}
$$

Здесь $c$ и $d$ - элементы $\mathfrak{K}$ (например, матрицы). Абелев вариант этой системы содержится в списках интегрируемых уравнений из работы [17]. 
ПримеР 3. Системы типа уравнения Бюргерса. Эти системы порождаются 2-градуированными алгебрами Ли и связаны с так называемыми левосимметрическими алгебрами [18]. Связь между левосимметрическими алгебрами и линеаризуемыми интегрируемыми системами отмечалась в работах $[5,14,19]$.

Пусть

$$
\mathfrak{B}=\left\{\left(\begin{array}{lll}
e & 0 & c \\
0 & f & d \\
0 & 0 & g
\end{array}\right)\right\}, \quad e, f, g, c, d \in \mathfrak{K}
$$

Зададим градуировку формулами

$$
\mathfrak{B}_{0}=\left\{\left(\begin{array}{ccc}
e & 0 & 0 \\
0 & f & 0 \\
0 & 0 & g
\end{array}\right)\right\}, \quad \mathfrak{B}_{1}=\left\{\left(\begin{array}{lll}
0 & 0 & c \\
0 & 0 & d \\
0 & 0 & 0
\end{array}\right)\right\}
$$

При этом остальные однородные компоненты равны нулю.

Выберем

$$
a=\left(\begin{array}{lll}
1 & 0 & 0 \\
0 & 1 & 0 \\
0 & 0 & 0
\end{array}\right), \quad \beta=\left(\begin{array}{lll}
\varepsilon & 0 & 0 \\
0 & \delta & 0 \\
0 & 0 & 0
\end{array}\right) \lambda^{2},
$$

где $\varepsilon, \delta$ - произвольные элементы из центра $\mathfrak{K}$.

Положим $\mathfrak{A}_{-}=\mathfrak{B}_{0}, \mathfrak{A}_{+}=\left\{q+S(q) \mid q \in \mathfrak{B}_{1}\right\}$ и

$$
S\left(\begin{array}{lll}
0 & 0 & c \\
0 & 0 & d \\
0 & 0 & 0
\end{array}\right)=\frac{1}{2}\left(\begin{array}{ccc}
-c-d+R(c+d) & 0 & 0 \\
0 & -c-d+R(c+d) & 0 \\
0 & 0 & c+d+R(c+d)
\end{array}\right)
$$

где $R$ - любое решение уравнения Янга-Бакстера (12).

Отметим, что операция

$$
X * Y=-X Y-Y X+[R(X), Y]
$$

задает на $\mathfrak{K}$ структуру левосимметрической алгебры. По-видимому, этот факт является новым и приводит к сериям интересных примеров левосимметрических алгебр.

Зададим оператор $L$ формулой (1), где $q \in \mathfrak{G}_{0}$, а оператор $A=A_{\beta}$ - формулой (11). Тогда уравнение Лакса $L_{t}=[A, L]$ эквивалентно неабелевой системе

$$
\begin{aligned}
c_{t}= & \varepsilon c_{x x}+2 \varepsilon(c+d) * c_{x}+(\varepsilon-\delta) d_{x} * c+ \\
& +(c+d) *((c+d) * \varepsilon c)-((c+d) *(\varepsilon c+\delta d)) * c, \\
d_{t}= & \delta d_{x x}+2 \delta(c+d) * d_{x}+(\delta-\varepsilon) c_{x} * d+ \\
& +(c+d) *((c+d) * \delta d)-((c+d) *(\varepsilon c+\delta d)) * d,
\end{aligned}
$$

где $c, d \in \mathfrak{K}$. Можно доказать, что это уравнение обладает высшими симметриями, не только если $*$ задана формулой $(25)$, но и в случае, когда $*$ является умножением в любой левосимметрической алгебре. Если $*$ - коммутативная ассоциативная операция, то приведенная выше система фактически содержится в [20]. По поводу ее обобшения на случай произвольного числа коммутируюших переменных см. [21]. 
ПримеР 4. Системы типа уравнения Буссинеска. Эти системы связаны с алгеброй Ли $\mathfrak{K}_{3}$ матриц третьего порядка над $\mathfrak{K}$.

Пусть $\mathfrak{B}=\mathfrak{K}_{3}$. Зададим градуировку формулами

$$
\mathfrak{G}_{3 i}=\left\{\left(\begin{array}{ccc}
e & 0 & 0 \\
0 & f & 0 \\
0 & 0 & g
\end{array}\right)\right\}, \mathfrak{G}_{3 i+1}=\left\{\left(\begin{array}{lll}
0 & e & 0 \\
0 & 0 & f \\
g & 0 & 0
\end{array}\right)\right\}, \quad \mathfrak{G}_{3 i+2}=\left\{\left(\begin{array}{lll}
0 & 0 & e \\
f & 0 & 0 \\
0 & g & 0
\end{array}\right)\right\} .
$$

Тогда $\mathfrak{G}=\bigoplus_{k \in \mathbb{Z}} \mathfrak{G}_{k} \lambda^{k}$.

Выберем

$$
\alpha=\left(\begin{array}{lll}
0 & 1 & 0 \\
0 & 0 & 1 \\
1 & 0 & 0
\end{array}\right) \lambda, \quad \beta=\left(\begin{array}{lll}
0 & 0 & 1 \\
1 & 0 & 0 \\
0 & 1 & 0
\end{array}\right) \lambda^{2}
$$

Рассмотрим случай $\mathfrak{A}_{+}=\mathfrak{G}_{0}, \mathfrak{A}_{-}=\{0\}$. Тогда уравнение $(2)$ - неабелева система

$$
\begin{aligned}
& q_{t}=\frac{i}{\sqrt{3}}\left(q_{x x}+\left[p_{x}, p\right]+[[p, q], q]\right)-\left(p^{2}\right)_{x}+\left[q^{2}, p\right], \\
& p_{t}=-\frac{i}{\sqrt{3}}\left(p_{x x}+\left[q_{x}, q\right]+[[q, p], p]\right)-\left(q^{2}\right)_{x}+\left[p^{2}, q\right],
\end{aligned}
$$

где $p, q \in \mathfrak{K}$. Эта система имеет симметрии порядков не кратных трем.

Пусть теперь

$$
\mathfrak{A}_{-}=\left\{\left(\begin{array}{lll}
f & 0 & 0 \\
0 & f & 0 \\
0 & 0 & f
\end{array}\right)\right\}, \quad \mathfrak{A}_{+}=\left\{\left(\begin{array}{ccr}
f+g+P(f)+S(g) & 0 & 0 \\
0 & f \varepsilon+g \varepsilon^{2}+P(f)+S(g) & 0 \\
0 & 0 & f \varepsilon^{2}+g \varepsilon+P(f)+S(g)
\end{array}\right)\right\} .
$$

Здесь $\varepsilon$ - первообразный корень третьей степени из единицы, $P$ и $S$ - отображения из $\mathfrak{K}$ в $\mathfrak{K}$ такие, что $\mathfrak{A}_{+}$- подалгебра Ли в $\mathfrak{G}_{0}$. Примером таких $P$ и $S$ являются операторы

$$
P(X)=-T X, \quad S(X)=-T^{2} X
$$

где $T^{3}=1, X \in \mathfrak{K}$.

Другой пример связан с решением $R$-модифицированного уравнения Янга-Бакстеpa (12):

$$
P(X)=\frac{\varepsilon+1}{1-\varepsilon} X+\frac{2}{\varepsilon-1} R(X), \quad S(X)=\frac{\varepsilon^{2}+1}{1-\varepsilon^{2}} X+\frac{2}{\varepsilon^{2}-1} R(X) .
$$

Соответствущее уравнение (2) представляет собой неабелеву систему

$$
\begin{aligned}
p_{t} & =-\left(A_{1}\right)_{x}+\left[A_{2}, q\right]+\left[P\left(A_{1}\right)+S\left(A_{2}\right), p\right]-\left[P(p)+S(q), A_{1}\right], \\
q_{t} & =-\left(A_{2}\right)_{x}+\left[A_{1}, p\right]+\left[P\left(A_{1}\right)+S\left(A_{2}\right), q\right]-\left[P(p)+S(q), A_{2}\right],
\end{aligned}
$$

где $A_{1}=-8 p_{x}+(\sqrt{3} i+3) q^{2}+8[p, P(p)+S(q)]$ и $A_{2}=8 q_{x}+(\sqrt{3} i+3) p^{2}-8[q, P(p)+S(q)]$. Выбирая в качестве $T$ в формуле $(26) 1, \varepsilon$ и $\varepsilon^{2}$, можно получить еше три конкретных примера неабелевых систем типа уравнения Буссинеска.

Мы благодарны Б. А. Дубровину за полезные обсуждения.

Исследования были выполнены при финансовой поддержке Российского фонда фундаментальных исследований (грант 96-01-00382) и INTAS (проект 93-166-EXT). 


\section{Список литературы}

[1] В. Г. Дринфельд, В. В. Соколов. В кн.: Итоги науки и техники. Современные проблемы математики. Т. 24. М.: ВИНИТИ, 1984. С. 81-180.

[2] A. P. Fordy, P. P. Kulish. Commun. Math. Phys. 1983. V. 89. P. 427-443.

[3] C. Athorne, A. P. Fordy. J. Phys. A. 1987. V. 20. P. 1377-1386.

[4] В. A. Марченко. Нелинейные уравнения и операторные алгебры. Киев: Наукова думка, 1986.

[5] S. I. Svinolupov. Phys. Lett. A. 1987. V. 135. P. 32-36.

[6] S. I. Svinolupov. Commun. Math. Phys. 1992. V. 143. P. 559-575.

[7] С. И. Свинолупов. Функц. анализ и его прилож. 1993. Т. 27. № 4. С. 40-53.

[8] С. И. Свинолупов, В. В. Соколов. ТМФ. 1994. Т. 100. № 2. С. 214-218.

[9] С. И. Свинолупов, В. В. Соколов. ТМФ. 1996. Т. 108. № 3. С. 388-392.

[10] M.F. de Groot, T. J. Hollowood, J. L. Miramontes. Commun. Math. Phys. 1992. V. 145. № 1. P. $57-84$.

[11] А. А. Белавин, В. Г. Дринфельд. Функц. анализ и его прилож. 1982. Т. 16. № 3. С. 1-29.

[12] М. А. Семенов-Тян-Шанский. Функц. анализ и его прилож. 1983. Т. 17. № 4. С. 17-33.

[13] И. З. Голубчик, В. В. Соколов. ТМФ. 1997. Т. 110. № 3. С. 339-350.

[14] V. V. Sokolov, S. I. Svinolupov. Acta Appl. Math. 1995. V. 41. P. 323-339.

[15] F. A. Khalilov, E. Ya. Khruslov. Inv. Prob. 1990. V. 6. P. 193-204.

[16] О. В. Мельников, В. Н. Ремесленников, В.А. Романьков, Л. А. Скорняков, И. П. Шестаков. Общая алгебра. Т. 1. М.: Наука, 1990.

[17] А. В. Михайлов, А. Б. Шабат, Р. И. Ямилов. УМН. 1987. Т. 42. № 4. С. 3-53.

[18] A. Medina. C. R. Acad. Sci. Paris, Ser. A. 1978. V. 286. P. 173-177.

[19] Б. А. Дубровин, С. П. Новиков. УМН. 1989. Т. 44. № 6. С. 29-98.

[20] В. В. Соколов. УМН. 1988. Т. 43. № 5. С. 133-163.

[21] С.И. Свинолупов, В. В. Соколов. УМН. 1992. Т. 47. № 3. С. 115-147.

Поступила в редакцию 13.II.1997 г.

\section{Z. Golubchik, V. V. Sokolov}

\section{INTEGRABLE EQUATIONS ON ZZ-GRADED LIE ALGEBRAS}

Evolution systems with $L-A$-pairs in $\mathbb{Z}$-graded Lie algebras are investigated. Some different hierarchies of integrable systems are associated with the same $L$-operator. They correspond to different decompositions of zero component of the $\mathbb{Z}$-graded algebra in a direct sum of two subalgebras. As the result, new examples of multi-component integrable systems are constructed. 